\title{
Simulation of phase transitions and strength evolution in quartzite in shock experiments
}

\author{
Mikhail Kovalenko, Denis Shalkovsky", Aleksandr Petrovtsev, Aleksandr Pavlenko, \\ Svetlana Malyugina, Dmitriy Yusupov \\ Russian Federal Nuclear Center - Zababakhin Institute of Applied Physics, Snezhinsk, Russia
}

\begin{abstract}
Quartzite is a basic rock constituent. It has a complicated phase diagram where besides a low-pressure phase ( $\alpha$-quartz), two high-pressure solid ones are present - coesite and stishovite. Also present are the hightemperature cristobalite and tridymite phases with very small stability regions. In shock experiments, the transition to coesite almost never realizes and the transition from $\alpha$-quartz to stishovite proceeds in a strongly nonequilibrium regime characterized by metastability and a long transition time. Quartzite is polycrystalline quartz with small amounts of impurities. The paper describes experiments with samples of Pervouralsk quartzite with mass fractions of $\mathrm{SiO}_{2}$ above $96 \%$.
\end{abstract}

\section{Equation of state}

The thermodynamic properties of quartzite are described by a multiphase EOS [1] which includes three main phases: alpha-quartz, stishovie, and liquid quartz. The position of the $\alpha$-quartz-stishovite equilibrium line (Figure 1) is determined from experiments with shocked quartzite unloading into buffer materials. The experiments were performed at RFNC-VNIITF by Zhugin et al [2]. The velocity of the quartzite-buffer boundary was measured with induction sensors. The points where stishovite transformed back to $\alpha$-quartz were determined from the breakpoints of unloading adiabats which were compared with calculated data.

Metastability of states on the quartzite Hugoniot is described with a polymorphous transition model based on a kinetic equation of relaxation type. Methods for constructing the multi-phase EOS with a nonequilibrium transformation is described in [12]. The metastable phase concentrations are specified as functions of the degree of state metastability defined by the Gibbs potential difference. The limiting phase concentrations corresponding to loading and unloading adiabats reproduce metastable and equilibrium transformations. The reverse transformation is described within a quasi-equilibrium approach. The transformation rate in the kinetic equation is defined by the characteristic transformation time.

\footnotetext{
*Corresponding author: d.m.shalkovskiy@mail.ru
} 

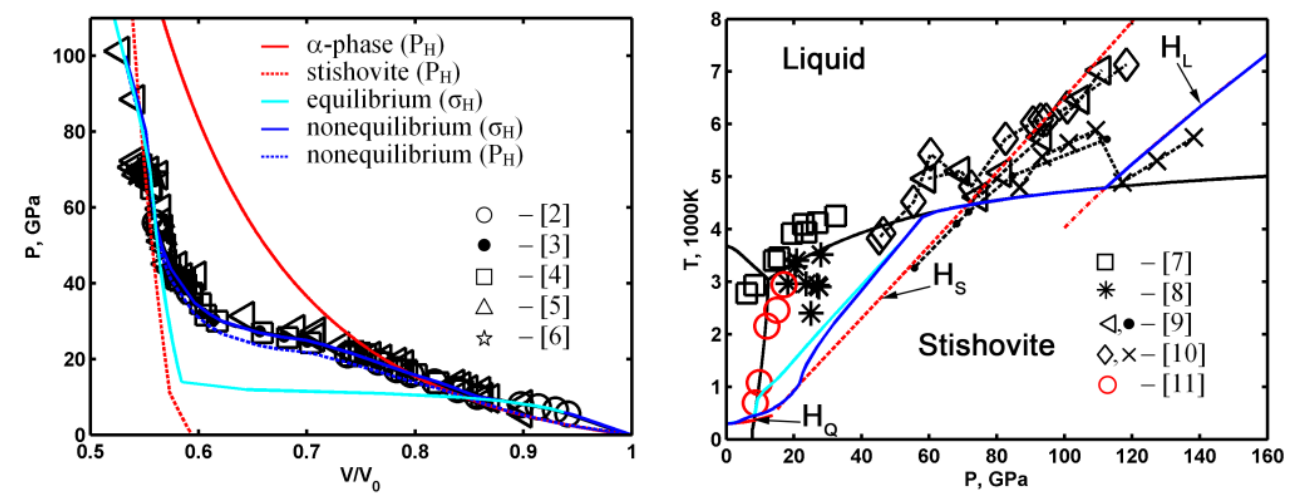

Fig. 1. Quartzite shock compressibility (left) and quartzite phase diagram from EOS [1] (right). Markers show experimental data, lines show calculated data.

\section{Elastic-plastic properties}

The multiphase EOS of quartzite is extended by constitutive relations describing the evolution of shear stresses versus phase composition. In the region of mixed phases, the yield stress and the shear modulus are determined from phase ratios. For pure phases, the yield stress and shear modulus with respect to plastic strain $\varepsilon_{\mathrm{p}}$ and thermodynamic state parameters (pressure $\mathrm{P}$ and temperature T) are determined using the Steinberg model [13]. Softening is taken into account by considering two branches of deformation corresponding to intact and failed materials with a linear transition between them upon reaching a certain value of plastic strain.

Figure 2 shows yield stress versus plastic strain in quartzite along its Hugoniot from calculations with adjusted model parameters. Shear stresses were evaluated through comparison between the "elastic-plastic" Hugoniot $\left(\sigma_{\mathrm{H}}\right.$ in Figure 1) of quartzite and its "hydrodynamic" Hugoniot $\left(\mathrm{P}_{\mathrm{H}}\right.$ in Figure 1) obtained in experiments with samples of ground quartzite mixed with high-plasticity paraffin and fluoroplastic [2,11]. This comparison allows us to infer that the shear component of stresses in quartzite under shock compression is rather high. Accordingly, yield stress initially increases to a maximum of $\approx 5 \mathrm{GPa}$ at $\varepsilon_{\mathrm{p}}=1 \%$ and then, as quartzite softens, decreases to $\approx 2 \mathrm{GPa}$ at $\varepsilon_{\mathrm{p}}=0.08$. Its further changes occur due to phase transformations.

\section{Experiments}

In experiments we investigated the behavior of monolithic quartzite from Pervouralsk under shock waves. The average density of quartzite targets was $2.639 \mathrm{~g} / \mathrm{cm}^{3}$. The quartzite sample ( $35 \mathrm{~mm}$ in diameter) was loaded by $12 \mathrm{Cr} 18 \mathrm{Ni10Ti}$ steel and aluminum flyers. Thick flyers $(5,6$, and $8 \mathrm{~mm}$ thick ones were used) are needed to allow registration of stationary flow sections in the velocity profiles and then determine the time when the phase transformation ends behind the front of the principle plastic wave. An explosive loading device (ELD) or a one-stage light-gas gun (LGG1) was used to accelerate the flyers. In one experiment, a two-stage light-gas gun (LGG2) was used. Details of the experimental setup are provided in Table 1.

In LGG1 experiments, mass velocity profiles on the boundary between the sample and the lithium fluoride (LiF) window were registered with the VISAR interferometric measurement technique. In the other experiments, the stress profile was measured with the manganin gauge technique (MGT). The gauge was mounted between parts of the cut 
sample (their thicknesses in the shock propagation direction are presented in Table 1) and then the sample with the gauges between its parts was put into a marble case in order to increase the registration time.

Table 1 - Shock experiments with quartzite samples

\begin{tabular}{|c|c|c|c|c|c|c|c|c|c|c|c|}
\hline \multirow[t]{2}{*}{ Test \# } & \multirow{2}{*}{$\begin{array}{l}\text { Registration } \\
\text { technique }\end{array}$} & \multirow{2}{*}{$\begin{array}{l}\begin{array}{l}\text { Loading } \\
\text { device }\end{array} \\
\end{array}$} & \multicolumn{3}{|c|}{ Flyer } & \multicolumn{3}{|c|}{ Sample } & \multirow{2}{*}{$\begin{array}{l}\text { Screen, } \\
\text { window }\end{array}$} & \multirow{2}{*}{$\begin{array}{l}\sigma_{1},{ }^{1} \\
\mathrm{GPa}\end{array}$} & \multirow{2}{*}{$\begin{array}{c}\xi_{\mathrm{s},}{ }^{2} \\
\%\end{array}$} \\
\hline & & & material & \begin{tabular}{|l|}
$\mathrm{h}_{\mathrm{f}}$ \\
$\mathrm{mm}$
\end{tabular} & \begin{tabular}{|l|}
$\mathrm{W}_{\mathrm{f}}$ \\
$\mathrm{km} / \mathrm{s}$
\end{tabular} & $\begin{array}{l}\mathrm{h}_{1}, \\
\mathrm{~mm}\end{array}$ & $\begin{array}{l}\mathrm{h}_{2}, \\
\mathrm{~mm}\end{array}$ & $\begin{array}{l}\mathrm{h}_{3}, \\
\mathrm{~mm}\end{array}$ & & & \\
\hline 1 & VIZAR & LGG1 $^{4}$ & $\mathrm{SS}^{3}$ & 6 & \begin{tabular}{|l|}
0.78 \\
\end{tabular} & 4 & & & $\mathrm{LiF}$ & 8.59 & 0 \\
\hline 2 & VIZAR & LGG1 & SS & 6 & 1.24 & 4 & & & $\mathrm{LiF}$ & 13.16 & 0.15 \\
\hline 3 & VIZAR & LGG1 & SS & 6 & \begin{tabular}{|l|}
1.24 \\
\end{tabular} & 8 & & & $\mathrm{LiF}$ & 13.08 & 0.15 \\
\hline 4 & VIZAR & LGG1 & SS & 6 & 1.59 & 4 & & & $\mathrm{LiF}$ & 17.08 & 10.35 \\
\hline 5 & VIZAR & LGG1 & SS & 6 & 1.51 & 8 & & & $\mathrm{LiF}$ & 16.16 & 8.00 \\
\hline 6 & VIZAR & LGG1 & SS & 6 & \begin{tabular}{|l|}
1.71 \\
\end{tabular} & 4 & & & $\mathrm{LiF}$ & 18.53 & 14.55 \\
\hline 7 & VIZAR & LGG1 & SS & 6 & \begin{tabular}{|l|l|}
1.67 \\
\end{tabular} & 8 & & & $\mathrm{LiF}$ & 18.00 & 12.72 \\
\hline 8 & MGT & LGG2 $^{5}$ & SS & 6 & 2.55 & 4 & 20 & & PMMA & 27.98 & 61.00 \\
\hline 9 & MGT & ELD $^{6}$ & $\mathrm{Al}$ & 8 & 3.56 & 6 & 8 & & PMMA & 29.74 & 70.72 \\
\hline 10 & MGT & ELD & $\mathrm{Al}$ & 8 & 3.95 & 4 & 4 & 8 & - & 34.10 & 81.77 \\
\hline 11 & MGT & ELD & $\mathrm{Al}$ & 8 & 4.47 & 4 & 4 & 8 & - & 40.86 & 89.64 \\
\hline 12 & MGT & ELD & SS & 4 & 3.60 & 6 & 8 & & PMMA & 43.91 & 92.52 \\
\hline 13 & MGT & ELD & SS & 4 & 3.90 & 6 & 8 & & PMMA & 48.58 & 96.92 \\
\hline $\begin{array}{ll}1 & \text { plate } \\
2 & \text { mass } \\
3 & 12 \mathrm{Cr} \\
4 & \text { LGG } \\
5 & \text { LGG } \\
6 & \text { ELD }\end{array}$ & $\begin{array}{l}\text { value behind } \\
\text { ction of extra } \\
\text { Ni10Ti stainle } \\
\text { one-stage lig } \\
\text { two-stage lig } \\
\text { explosive load }\end{array}$ & $\begin{array}{l}\text { the shock } \\
\text { icted stish } \\
\text { ess steel } \\
\text { ht-gas gur } \\
\text { ht-gas gu } \\
\text { ling devic }\end{array}$ & $\begin{array}{l}\text { front } \\
\text { vite behin flyer dia } \\
\text {, flyer dia }\end{array}$ & ad the & $\begin{array}{l}\text { shock } \\
\text { r } 34 \mathrm{mn} \\
\text { r } 50 \mathrm{mn}\end{array}$ & front & & & & & \\
\hline
\end{tabular}

\section{Calculations}

Calculations presented in the paper were done by the 1-D VOLNA code [14]. Figure 3 compares calculated and experimental velocity profiles for LGG1 loaded quartzite samples. The calculation reproduces the basic features of the velocity profile: elastic precursor separation; the signal characteristic of softening on the shock front; the initial stage of phase precursor separation. By the example of calculations for Test 7 with the equilibrium phase transition and without it (the $\alpha$-phase only), phase transformation kinetics is shown to play a key role in the formation of the stress profile in the sample if even the level of stishovite production is low. The level of stishovite production defines the evolution of the shock front propagating through the sample thickness, specifically, separation and time lag between the elastic/phase precursor and the principal wave.

Our simulations suggest that a small change in the plastic strain threshold for $\alpha$-quartz, at which softening begins, greatly influences the structure of the shock front (changing the amplitude of the softening signal and its position between the elastic precursor and the plastic wave). The threshold value is not easy to determine because of inhomogeneity in quartzite properties from sample to sample. From a series of calculations we determined an average value which helped get the shock profile close to experiment.

On the velocity profiles of 4-mm-thick samples, the following typical sections can be identify behind the shock front: the steady flow plateau, the local decrease, and then the smooth increase till the time when the rarefaction wave arrives. Our analysis of calculated results suggests that the local decrease is caused by the circulation of the rarefaction wave formed due to the reflection of the elastic precursor from the window. A similar local decrease is present on the calculated velocity profiles too, but it is not that expressed in 
amplitude. The further smooth increase of velocity is caused by plastic wave circulation in the sample "squeezed" between the window and the flyer. Each wave passage increases pressure and stishovite production which causes wave smoothing. Note that a similar increase of velocity can also be seen in the calculated velocity profile. Registration ends simultaneously with the failure of the window in calculation. We suppose the sharp decrease of velocity before the end of registration in tests 1 and 2 is caused by the failure of the window rather than by processes in the sample.

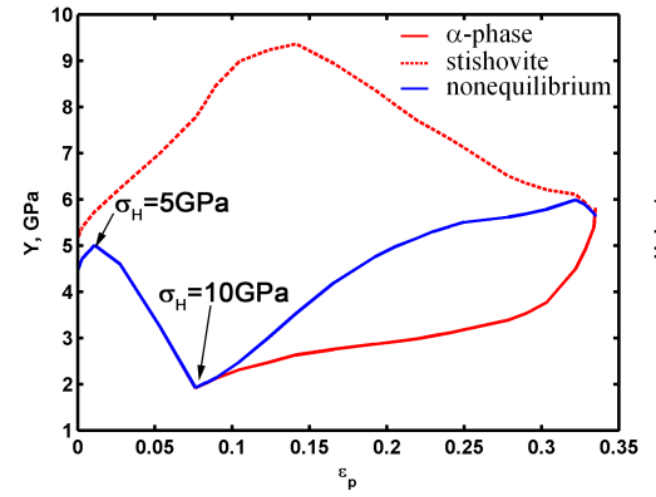

Fig. 2. Yield stress versus plastic strain along the Hugoniot.

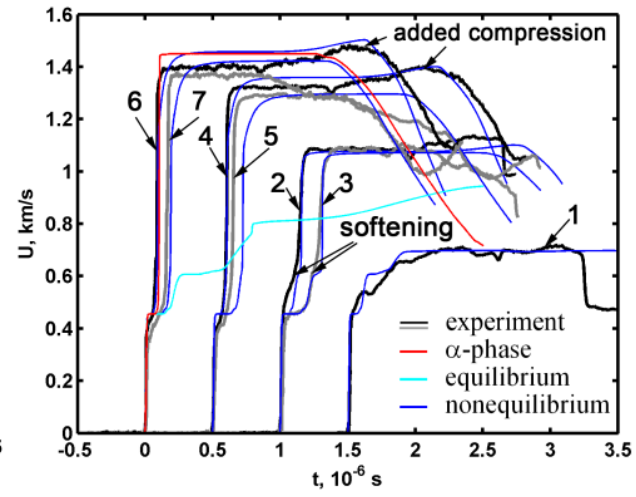

Fig. 3. Velocity profiles on the sample-window boundary in LGG1 tests.

In experiments with 8-mm-thick samples, the section of added compression is absent because of the following reasons: due to the larger thickness the rarefaction wave arrives before the compression wave finishes its circulation in the sample, and, according to our evaluation, in tests 3,5 , and 7 , the effect of lateral unloading manifests itself within $\Delta \mathrm{t} \sim 1 \mu \mathrm{s}$ after the elastic precursor arrives and shock front registration starts.

Figure 4 shows stress profiles from manganin gauge measurements in tests 8-13. Because of the relatively small sample thickness and technique peculiarities, it is difficult to identify the elastic precursor and the time when the phase precursor and the plastic wave separate. Note that the experimental stress profiles show the registered unloading with a characteristic signal from the reverse stishovite- $\alpha$-quartz transition.

In ELD and LGG2 experiments, a higher level of loading realizes in samples (shock wave amplitudes 30-50 GPa). As a result, stishovite production is rather high $(60-95 \%$; see Table 1 for more detail). Calculations with the initial model used to describe tests with stishovite production below $15 \%$ do not reproduce the shock front profile. To improve the reproduction of the experimental profiles at high levels of loading, we had to modify the description of phase transition kinetics.

Comparison between calculations with different descriptions of $\alpha$-quartz-stishovite transition kinetics and experimental results (Figure 4) shows that both models reproduce stress amplitudes in the steady flow section which points to acceptable accuracy in compressibility and stishovite production calculation. Calculations of tests 10 and 11 reproduce well diversity in the time of shock arrival at gauges at different sample thicknesses.

Calculations with the direct phase transition kinetics model parametrized so as to reproduce high-level loading experiments give a qualitative description of the shock front and show better agreement between calculation and experiment in the unloading section. They reproduce well the time when the rarefaction wave arrives from the free surface of the flyer and the further decrease of stresses which proves that the longitudinal sound velocity is described correctly. In the unloading section of stress profiles calculated for tests 12 and 13, one can clearly see a step formed due to the reverse phase transition. On the 
experimental profiles, the step is not that sharp possibly because of the effect of lateral unloading or the nonequilibrium character of the reverse transition (which is equilibrium in calculation).
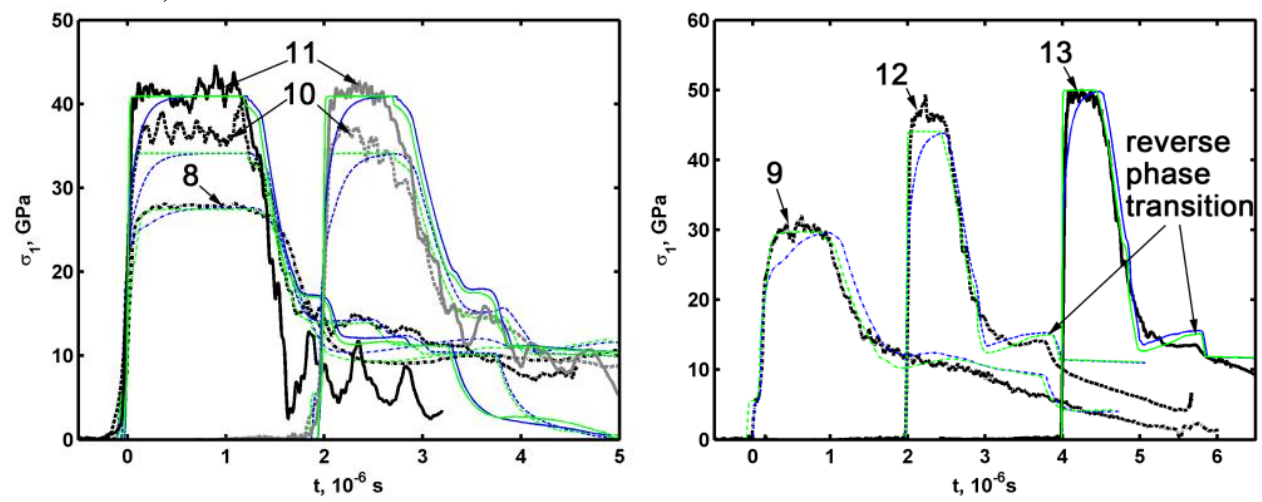

Fig. 4. Experimental stress profiles from manganin gauge measurements in comparison with calculations with different descriptions of $\alpha$-qurtz-stishovite transition kinetics. The right figure shows profiles measured in one test at distances 4 and $8 \mathrm{~mm}$ from the loading surface; the left is for a sample thickness of $6 \mathrm{~mm}$ before the gauge. The time interval between the profiles is artificially increased for convenience: the profiles are shown at a step of $2 \mu$ s beginning from the time of shock arrival. Experimental profiles are shown by black and gray lines. Calculated profiles are shown in blue and green for different models of phase transition kinetics.

Possibly, in order to describe experiments with samples of small thicknesses (below 8 $\mathrm{mm}$ ) we will have to not only adjust model parameters, but also modify the model itself. Currently its limiting surface of metastable states does not vary with time thus allowing the wave structure to relax fast and earlier to stationary.

\section{Conclusion}

We have discussed results we obtained in our simulation of experiments aimed to study phase transitions in quartzite under shock loading. The model we used for calculation allows for the nonequilibrium regime of the direct $\alpha$-quartz-stishovite transition and the reduction of shear strength at low plastic strains, which is typical for rocks. Simulation of experiments at high levels of loading $\left(\sigma_{\mathrm{H}}>30 \mathrm{GPa}\right)$ suggests that possibly the reverse transition proceeds in the nonequilibrium regime too. In addition to available data we need similar results from experiments with thicker samples in order to allow further improvement of our model. In the future experiments with the use of interferometric techniques, it is desirable to provide for multi-channel registration in order to collect static data on the magnitude of the elastic precursor and the threshold value of strain at which softening begins.

\section{References}

1. A.V. Petrovtsev, V.V. Dremov, V.G. Vildanov, M.M. Gorshkov, V.T. Zahikin, Yu.N. Zhugin, AIP Conference Proceedings, 849, 380-392 (2006)

2. Yu.N. Zhugin, K.K. Krupnikov et al, in Proceedings of the USSR Academy of Sciences, Physics of the Earth, 10, 16-22 (1994) 
3. Yu.N. Zhugin, K.K. Krupnikov et al, in Proceedings of IUPAP-IUTAM Symposium "Problems in Nonlinear Acoustics", edited by E.G. Kedrinsky, Novosibirsk, Siberian Branch of USSR Academy of Sciences, 2, 196-200 (1987)

4. R.F. Trunin, G.V. Simakov et al, in Proceedings of the USSR Academy of Science, Physics of the Earth, 1, 13 (1971)

5. Chhabildas, L.C. Shock Loading and Release Behaviour of X-Cut Quartz. in Proceedings Shock Waves in Condensed Matter -1985, edited by Y.M. Gupta (Plenum Press, New-York), 601-605 (1986)

6. LASL Shock Hugoniot Data (Berkly, Los Angeles, London: University California Press, 1980)

7. R.J. Hemley, Reviews in Mineralogy, 29, 41-82 (1994)

8. K.I. Kondo, T.J. Ahrens, J. Appl. Phys., 54, 4383-4385 (1983)

9. R.G. McQueen, J.N. Fritz, J.W. Hopson, in Proceedings New ways for looking for phase transitions at multi-megabar dynamic pressures. Physics of solids under high pressures, 99-108 (1981).

10. G.A. Lyzenga, T.J. Ahrens, A.C. Mitchell, J. Geoph. Res., 88, No.33, 2431-2444 (1983).

11. Zhugin, Yu. N. The behaviour of $\alpha$-Quartz under High Dynamic and Static Pressures: New Results and Views, in Proceedings Shock Compression of Condensed Matter1995, Woodbury, New-York: AIP Press, Part I, 97-100 (1996).

12. V.V.Dremov, A.L.Koutepov, A.V. Petrovtsev, A.T. Sapozhnikov, AIP Conference Proceedings, 620. Ed. by M. Furnish, 87-90 (2001)

13. D.J.Steinberg, S.G.Cohran, M.W.Guinan. J. Appl. Phys., 51, 1498-1504 (1980).

14. V.F. Kuropatenko, G.V. Kovalenko et al, J. VANT, Mathematical Modeling of Physical Processes Series, Issue 2, 9-25 (1989) 